\title{
Cervical Cancer: The Trending Topic in Oncology
}

\section{Jerzy Krupinski*}

Department of Clinical Neurology, John Radcliffe Hospital, UK

Cervical cancer is malignant Carcinoma type of cancer originates in cervix region. It is a type of cancer that starts in the cervix i.e., the tight opening into the uterus from the vagina. The ordinary "ectocervix" (the bit of the uterus reaching out into the vagina) is solid pink shading and is secured with level, thin cells called squamous cells. The "endocervix" or cervical channel is comprised of another sort of cell called columnar cells. The territory where these phones meet is known as the "change zone" (T-zone) and is the undoubtedly area for irregular or precancerous cells to create.

Most cervical cancers are squamous cell carcinomas, emerging in the squamous (smoothed) epithelial cells that line the cervix. Adenocarcinoma, emerging in glandular epithelial cells is the $2^{\text {nd }}$ most regular sort. Rarely, cancer can emerge in different sorts of cells in the cervix. Tumor alludes to a class of ailments in which a cell or a gathering of cells partition and reproduce wildly, encroach into adjoining cells and tissues (intrusion) and at last spread to different parts of the body than the area at which they emerged (metastasis) (National Cancer Institute 2009).

Human papillomavirus (HPV) infection has every earmarks of being required in the change of over $90 \%$ of cases; by far most who have had HPV infections, in any case, don't make cervical development. Other related dangerous components involves smoking, a feeble safe structure, counteractive action pills, starting sex at a young age, and having various sexual associates, yet these are less fundamental. Cervical infection routinely starts from precancerous changes more than 10 to 20 years. Around $90 \%$ of cervical cancer cases are squamous cell carcinomas, $10 \%$ are adenocarcinoma, and somewhat number are distinctive sorts. Assurance is routinely by cervical screening took after by a biopsy.

Endometrial tumor is the growth of abnormal cells in the covering of the uterus. The covering is known as the endometrium. Endometrial tumor is also called sickness of the uterus, or uterine growth.

Checking the cervix by the Papanicolaou test, or Pap spread, for cervical infection has been associated with definitely diminishing the amount of occurrences and mortality from cervical tumor in most of the countries. Pap spread screening at normal interims with fitting follow-up can diminish cervical infection rate up to $80 \%$. Uncommon outcomes may propose the proximity of precancerous changes, allowing examination and possible preventive treatment. The treatment of worthless wounds may inimically affect productivity and pregnancy.

The treatment of cervical illness varies all over the world, all things considered, on account of access to authorities talented in radical pelvic surgery, and the ascent of "fertility-sparing therapy" is started in nations. Since cervical infections are radiosensitive, radiation may be used as a piece of all stages where surgical options don't exist. Microinvasive diseases (organize IA) may be managed by hysterectomy (removal of the whole uterus including some portion of the vagina). For stage IA2, the lymph center points are emptied, as well.

Early stages such as (IB1 and IIA under $4 \mathrm{~cm}$ ) can be cured with radical hysterectomy with departure of the lymph center points or by radiation treatment. Radiation treatment is given as external shaft radiotherapy to the pelvis and brachytherapy (inward radiation). Women cured with surgery who have high-chance segments found on pathologic examination are given radiation treatment with or without the use of chemotherapy to decrease the risk of descend into sin.

Nowadays uterus cancer disease is most common in females affecting the genital tract characterized by cervical cancer, with an increasing incidence in the last decades. As per the records of the world health organization (WHO Report 2015), cervical cancer is declared as the world's second fatal cancer since it causes 273,505 deaths per year including 493,243 women diagnosed with it every year. Cervical cancer deaths occur most frequently among women aged between 15 and 44 years. The causes which lead to these cancers are the average lifespan increase in the female population above 65 years of age, and the existence of some risk factors such as unopposed estrogen replacement therapy for menopausal women, polycystic ovary disease, obesity, etc. Endometrial cancer is the second most common cancer causing death next to cervical cancer. However, it is the third most common cause of death among the female genital cancers next to ovarian and cervical cancers.
*Corresponding author: Jerzy Krupinski, Professor, Department of Clinical Neurology, John Radcliffe Hospital, UK, Tel: +44 300304 7777; E-mail: jamesbruiners.uk@rediffmail.com

Received June 22, 2017; Accepted June 26, 2017; Published June 29, 2017

Citation: Krupinski J (2017) Cervical Cancer: The Trending Topic in Oncology. J Vasc Med Surg 5: e120. doi: 10.4172/2329-6925.1000e120

Copyright: (c) 2017 Krupinski J. This is an open-access article distributed under the terms of the Creative Commons Attribution License, which permits unrestricted use, distribution, and reproduction in any medium, provided the original author and source are credited. 